\title{
LAS PUGNAS DE LAS MADRES \\ COMUNITARIAS POR NOMBRAR \\ EL TRABAJO DE CUIDADOS ${ }^{1}$
}

Adriana De León ${ }^{2}$

THE STRUGGLES OF COMMUNITY MOTHERS FOR

THE RECOGNITION OF CARE WORK

AS LUTAS DAS MÃES COMUNITÁRIAS PELO

RECONHECIMENTO DO TRABALHO DE CUIDADOS

Fecha de recepción: 30 de noviembre del 2020

Fecha de aceptación: 15 de abril del 2021

Disponible en línea: 4 de junio del 2021

Sugerencia de citación. De León, A. (2021). Las pugnas de las madres comunitarias por nombrar el trabajo de cuidados. Razón Crítica, (11), 65-95. https://doi.org/10.21789/25007807.1763

(1) El presente artículo es resultado del trabajo de maestría El género de las políticas públicas: las pugnas de las madres comunitarias por nombrar el trabajo de cuidados, presentado para obtener el título de Máster en Estudios Avanzados del Trabajo y el Empleo en la Universidad Complutense de Madrid.

(2) Abogada, especialista en Estudios Feministas y de Género, master en Estudios Avanzados del Trabajo y el Empleo | hadina.loi@gmail.com 


\section{R E S U M E N}

El escaso valor atribuido al trabajo de las mujeres se remonta a la división sexual de las actividades entre el espacio productivo y el reproductivo, bajo un criterio de valoración del mercado que constituye un índice semántico para considerar lo que es trabajo y lo que no. Esta dualidad valorativa se ha trasladado a las actividades remuneradas, en las que el género ha sido el criterio de diferenciación para ordenar y dar estatus a los trabajos pagados. En Colombia, al trabajo de los cuidados se le han asignado cualidades morales que lo convierten en actividades voluntarias, desinteresadas y gratuitas. Por ello, este artículo se propone hacer un análisis crítico, desde una perspectiva feminista, sobre la reivindicación de los derechos de las mujeres en la disputa por nombrar y reconocer su trabajo. Se ilustrará el caso de las madres comunitarias en los cuidados de la niñez al servicio del Estado, explorando los criterios interpretativos de la Corte Constitucional en los últimos treinta años sobre los cuidados como trabajo y la realidad laboral de las cuidadoras.

PALABRAS CLAVE: Género; trabajo de los cuidados; trabajo productivo; madres comunitarias. 


\section{A B S T R A C T}

The modest value assigned to women's work dates back to the sexual division of activities between the productive and reproductive dimensions under market valuation criteria that constitute a semantic index of what is considered work and what is not. This duality has been transferred to paid work, in which gender has been the differentiating criterion for ordering and giving status to paid jobs. In Colombia, care work has been granted moral features that make it a set of voluntary, selfless, and non-paid activities. For this reason, this paper makes a critical analysis, from a feminist perspective, on the vindication of women's rights in the dispute for the recognition of their work as caregivers. The case of community mothers responsible for children care at State service centers will be depicted, exploring the interpretive criteria by the Constitutional Court during the last thirty years on care as work and the labor reality of caregivers.

Keywords: Gender; care work; productive work; community mothers.

\section{R E S U M O}

O escasso valor atribuído ao trabalho das mulheres remonta à divisão sexual das atividades entre o espaço produtivo e o reprodutivo, sob um critério de valorização do mercado que constitui um índice semântico para considerar o que é trabalho e o que não. Essa dualidade valorativa passou às atividades remuneradas, nas quais o gênero tem sido o critério de diferenciação para organizar e dar status aos trabalhos remunerados. Na Colômbia, ao trabalho dos cuidados, são designadas qualidades morais que o convertem em atividades voluntárias, desinteressadas e gratuitas. Por isso, neste artigo, propõe-se fazer uma análise crítica, de uma perspectiva feminista, sobre a reivindicação dos direitos das mulheres na disputa pela nomeação e pelo reconhecimento de seu trabalho. É ilustrado o caso das mães comunitárias nos cuidados da infância para o serviço do Estado, explorando os critérios interpretativos da Corte Constitucional nos últimos 30 anos sobre os cuidados como trabalho e a realidade trabalhista das cuidadoras.

PALAVRAS-CHAVE: gênero; trabalho dos cuidados; trabalho produtivo; mães comunitárias. 
El trabajo de cuidados realizado por las mujeres ha sido infravalorado. En la producción discursiva dominante sobre las relaciones económicas, se han asentado criterios de valoración que invisibilizan el trabajo reproductivo dentro de la categoría de trabajo. Así, la producción doméstica, cuyo sujeto es femenino, es definida como trabajo privado, de modo que se la aísla de la producción social. Esta desvalorización se extrapola a la esfera pública del trabajo remunerado, con lo cual el trabajo de cuidados adquiere un estatus menor en una estructura social jerarquizada en función del sexo y, por ende, se justifica la percepción de salarios desiguales.

Si bien la Constitución política de 1991 dio lugar a la democratización de libertades individuales y a la consagración explícita de importantes derechos con los que se reconoció a las mujeres como sujetos plenos, también consolidó, sin embargo, el proyecto neoliberal implementado en Latinoamérica, autorizando principalmente las libertades económicas y la privatización (Burgos et al., 2009). Con ello, “[...] el peso pesado de las reformas económicas neoliberales cayó fuertemente sobre las mujeres de clases bajas e indígenas empobrecidas" (Paredes, 2008, p. 4).

En este contexto, Colombia se configuró como un régimen laboral precario y de informalidad creciente, con unas políticas sociales fragmentarias y focalizadas solo en ciertos sectores, que se basan más en el asistencialismo que en los derechos, dejando el arduo trabajo de las familias para la gestión de riesgos como la práctica de una nueva "arquitectura de bienestar" (Martínez, 2007, 2008), que suple los vacíos del Estado y de los mercados laborales a partir de una importante feminización de las responsabilidades del cuidado.

Frente a este panorama, los movimientos de mujeres han luchado, en el marco constitucional, por nombrar y reconocer los derechos en relación con el género, valiéndose del feminismo de los últimos 30 años, que ha 
incidido en el discurso jurídico al cuestionar los esencialismos en función del sexo y causar rupturas en las categorías básicas del derecho, como la tradicional división sexual entre el trabajo productivo y el reproductivo.

Por esta razón, en este artículo se analizarán los cuidados, y los criterios de su valoración, reflejados en el tratamiento dentro del mercado de trabajo y la política social del Estado, como trabajo femenino al servicio de la producción de bienestar. En este sentido, se pregunta por el largo recorrido de las reivindicaciones de los derechos de las mujeres a través de los jueces constitucionales y sus interpretaciones, en clave de género, de las categorías jurídicas a la hora de nombrar y reconocer el trabajo de los cuidados al servicio del Estado.

Para ello, primero, se analizará la categoría género y la organización del trabajo de las mujeres; segundo, se hará una aproximación a los cuidados como trabajo y a la identidad de las cuidadoras; tercero, se indagará sobre el tratamiento de los cuidados en Colombia, y, cuarto, se problematizarán las categorías con las que se abordan los asuntos de género en materia de derechos de las mujeres, a través de la pugna de las madres comunitarias por el reconocimiento de su trabajo al servicio del Estado en los últimos 30 años.

\section{El género como orden social del trabajo de las mujeres}

\section{El género y las relaciones de poder}

El trabajo dirigido a la satisfacción de las necesidades básicas de subsistencia, a la producción compartida y a las actividades reproductivas se encuentra ya en las sociedades precapitalistas. En estas, las mujeres participaban y disponían de los bienes producidos y de la tierra; trabajaban fuera de casa y existía la labor cooperativa, de base familiar, entre padre, madre e hijas. Esto no quiere decir que no existieran relaciones asimétricas, pero sí ayuda a entender que la posición de las mujeres no puede tratarse como una realidad estática (Federici, 2015). Tras los procesos de acumulación y producción para el intercambio, una nueva lógica económica marcó la orientación y conceptualización de la categoría trabajo, y, con ello, a algunas actividades comprendidas en el espacio semántico de este término le fueron asignadas un reconocimiento de valor. Esto fundamentó un orden social basado en la transformación y disciplinamiento social de las labores de hombres y mujeres.

De acuerdo con lo anterior, se puede pensar que el orden social se establece por medio de relaciones de poder, entendiendo el poder, a 
partir del pensamiento foucaultiano, como las formas de dominio en una compleja relación de fuerzas y dispositivos que disciplinan y ejercen control a través de la construcción de regímenes de verdad (el saber). Tales formas de dominación y de regímenes de poder/saber son lo que el pensamiento crítico feminista pretende revelar al ubicar la posición de las mujeres en un sistema de relaciones de dominación y explotación llamado patriarcado (Campillo, 2003; Fuente, 2015).

El cuerpo hace parte del campo político y las relaciones de poder operan sobre él, lo dominan, lo disciplinan y modelan, exigiéndole unas formas, un trabajo, un comportamiento, etc. (Foucault, 2002). Este sometimiento no se da exclusivamente a través de instrumentos coercitivos violentos o ideológicos directos, sino que puede obrar de manera sutilmente organizada, existe como un "saber del cuerpo", que Foucault define como tecnología política del cuerpo, la cual se manifiesta en la construcción de las subjetividades y las relaciones asimétricas entre los sexos. Ese saber del cuerpo toma a su cargo el gobierno de la vida de las mujeres por medio de la dominación de una disciplina que se adopta en su propia existencia.

Así, la presencia de un discurso de legitimación de poder basado en la diferencia biológica de los sexos es explorada por el feminismo a través de la categoría de género. Esta categoría es un elemento constitutivo de las relaciones sociales, pues señala la construcción de patrones culturales de interpretación que configuran órdenes sociales jerarquizados entre lo femenino y lo masculino. El género, además, es una forma primaria de articular relaciones de poder, en la que, según Bourdieu (citado en Scott, 2003), "se estructuran la percepción y la organización, concreta y simbólica, de toda la vida social” (pp. 292-293).

Para Nancy Fraser (2006), el género induce a una diferencia social bidimensional que combina características de clase explotada y sexualidad despreciada. Asimismo, el género sirve como principio ordenador de la estructura económica de la sociedad capitalista, pues organiza la división fundamental entre el trabajo remunerado "productivo" y el no remunerado "reproductivo" — este último asignado principalmente a las mujeres como responsabilidad primaria-y supone una codificación de patrones culturales de interpretación y evaluación que asigna estatus sociales $^{3}$ (Fraser \& Honneth, 2006). Esto implica que el capitalismo no

3 Nancy Fraser adopta la concepción weberiana de estatus como la representación del poder 
puede reducirse a explicaciones en términos puramente económicos, sino que debe ser considerado como un orden social complejo, caracterizado por relaciones sociales de dominación y explotación, lo que traduce la construcción social y clasificación del trabajo reproductivo como un eje de opresión y desigualdad de género (Arruza, 2014).

En otras palabras, el género y otros ejes de subordinación, como la clase y la raza, articulan relaciones de poder que se estructuran en órdenes sociales como el económico y pueden operar a través de diferentes instituciones (por ejemplo, el derecho) para la división del trabajo, incidiendo en la subordinación y valoración del trabajo femenino.

\section{El trabajo de las mujeres: del sustento familiar al no trabajo}

La evolución del concepto de trabajo transcurre a lo largo de tres épocas. Entre los siglos XVIII y Xıx, "cada una va a agregar una capa de significación suplementaria, sin nunca substituirse a las anteriores" (Méda, 2007, p. 21). Hannah Arendt (1993) señala que Locke descubre el trabajo como fuente de propiedad; Adam Smith, como origen de riqueza, y Marx, como expresión de la humanidad del hombre. Indiscutiblemente, la génesis de las sociedades basadas en el trabajo supuso poner en el centro de la organización social las actividades "productivas" mediadas por el mercado, contribuyendo así a la invisibilización del trabajo de las mujeres. Entonces, si el concepto trabajo es una invención, la clasificación y diferenciación entre lo productivo e improductivo también lo es; no son categorías ni objetivas ni neutrales respecto al género.

De ahí en adelante, las mujeres son absorbidas por la institución de la unión conyugal, y la familia se convierte en parte de un régimen doméstico completamente privado, separado de la esfera pública y del espacio de la deliberación política, cuyo legado hoy mantiene muchos de sus cimientos. A esa disociación entre la familia y lo público se suma una mutación económica: el hogar deja de ser un lugar de producción (Fraisse, 2003, p. 20). Lo relevante de este problema es la definición de las mujeres como no trabajadoras, con lo cual se transforma el criterio de su valoración. 
A pesar de la separación de esferas, lo cierto es que las mujeres nunca se han mantenido completamente fuera del trabajo remunerado, sobre todo en aquellas situaciones que las impelía, por necesidad, a formar parte de la mano de obra asalariada, siempre peor remunerada que en el caso de los varones. Durante la industrialización, muchas actividades eran desarrolladas por mujeres; lo específico del proceso de instauración de este modelo económico es que estas actividades se desvalorizaron.

Para Luz Gabriela Arango (1994),

no es tanto lo "femenino" o "masculino" de las habilidades lo que determina su mayor o menor valoración sino el hecho de que las desempeñen mujeres u hombres, [lo que] aparece es una valoración desigual y culturalmente variable de las destrezas, habilidades o calificaciones según estas sean ejercidas por hombres o mujeres (pp. 48-49).

La consolidación contemporánea del sistema económico se ha basado en procesos sociales y discursivos de diferenciación y devaluación, centrando en lo productivo una perspectiva universal que invisibiliza el espacio de reproducción como categoría económica y de trabajo. Esta desvalorización se transfiere a la esfera pública del trabajo remunerado, con lo cual se estable una discriminación en el empleo y el salario.

\section{Aproximación al trabajo de los cuidados}

\section{Los cuidados como trabajo}

Situar los cuidados como actividad y trabajo ha sido una aproximación propia de los debates feministas, que, desde distintas disciplinas, han trazado la construcción de la categoría. Estos enfoques coinciden en cuestionar los conceptos hegemónicos, en particular de la economía, acerca de la producción y el trabajo, que ocultan y devalúan la producción de bienes y servicios llevados a cabo por mujeres fuera de la economía de mercado.

Una definición concreta de los cuidados puede limitar sus alcances, porque es una categoría abierta, en permanente construcción y en disputa. Esta actividad se materializa en la producción diaria de bienestar físico y emocional en las personas, que va desde el cuidado directo (por ejemplo, alimentar a alguien) hasta el cuidado indirecto (hacer las compras, preparar alimentos, etc.), y que requiere de una gestión mental, como planificar cuándo y dónde hacer las compras (Pérez Orozco, 2017). En este sentido, 
Marx reclamaba que el trabajo debía ser una actividad liberadora y humanizante. Se trata de promover que la producción y, por tanto, el trabajo intervengan no solo en la mejora de la calidad de vida, sino también en la plena realización del individuo y de la sociedad (Méda, 2007). Sin embargo, ese mundo solo es posible gracias al otro oculto de la producción directa de la vida y de su sostenimiento, de los afectos, de las relaciones más íntimas, esto es, el mundo de los cuidados (Serrano \& Prieto, 2013).

En este sentido, el trabajo de los cuidados dentro del mercado laboral ha revelado cuánto aporta al bienestar general y al desarrollo económico, hecho que despertó el interés por analizar el trabajo de los cuidados en los campos profesionales y los servicios domésticos remunerados. En esa medida, las disputas por el significado del trabajo se impulsan con la presencia de nuevos actores sociales, como el movimiento feminista. Por ello, el trabajo de los cuidados hace parte de la economía global y ha sido reconocido por la oIT como promotor de uno de los mercados más dinámicos en los tiempos de crisis. Este trabajo presenta una doble cara: por un lado, es una actividad que tiene una demanda de mercado que va en aumento ${ }^{4}$, convirtiéndose "en una verdadera economía de mercado"; y, por otro, es una actividad dentro de la cual se reproducen desigualdades de género, clase y raza al entrar en el ciclo mercantil, como sucede con las cadenas globales de cuidados (Fraser, 2014; Martín Palomo, 2011, p. 90).

De acuerdo con lo anterior, Martín Palomo (2011) hace énfasis en la actual transformación del mundo laboral, en el que las fronteras del trabajo/no trabajo se desdibujan, primero, porque los trabajos son fragmentarios y dispersos, abarcando el espacio doméstico (como sucede con el teletrabajo); y, segundo, porque se caracteriza por una flexibilización y precarización de las condiciones laborales. Estos cambios, que deslocalizan, desagregan y rompen las formas de gestionar los procesos productivos y la vinculación de las personas, obligan a buscar nuevas herramientas conceptuales, razón por la cual propone una reflexión sobre el trabajo desde el término domesticación, como un modo de sustituir el término feminización, con el objetivo de descentrar la dualidad femenino/masculino y ubicar el análisis en las condiciones y los ámbitos en los que opera el trabajo y no en función del sujeto que lo

4 Según datos de la oit (2018, p. 2), en 2015, existían 2.100.000.000 de personas necesitadas de cuidados. Se prevé que, en el 2030, el número de beneficiarios de cuidados ascenderá a 
realiza. En consecuencia, Martín Palomo (2011, p. 70) plantea abordar los cuidados a partir de tres premisas: 1) revisión histórica del concepto del trabajo construido desde la división sexual; 2) reconceptualización de este para adaptarlo a los cambios sociales, y 3) recuperación del valor social de los cuidados, en términos de tiempo y dinero y en sentido ético. Con ello, su propuesta conceptual pretende sustraer el significado peyorativo del término doméstico porque el espacio doméstico es el que más ha sido transitado por las mujeres. Para la autora, "los cuidados son sobre todo trabajos, prestados con/por amor, por dinero o a cambio de cualquier otro tipo de bienes materiales o simbólicos, pero trabajo al fin de cuentas [...]" (Martín Palomo, 2011, p. 75).

Lo anterior demanda un cambio de perspectiva de las diferentes disciplinas sobre las nociones de trabajo y cuidados, sobre todo, cuando el modelo económico existente empuja a que los cuidados sean indispensables para la producción económica.

\section{LA IDENTIDAD DE LAS CUIDADORAS EN AMÉRICA LATINA}

En América Latina hay unos mandatos culturales impuestos a las mujeres en dos sentidos: el maternalismo y el reforzamiento de vínculos de solidaridad (voluntariado), que inciden en la manera como ha sido asumido tradicionalmente el trabajo de los cuidados.

El maternalismo ha tenido importantes efectos en la distribución de las responsabilidades familiares y la representación del cuidado. La imposición de esta identidad se fundamenta en una supuesta atribución de valores considerados específicos de las mujeres, lo que provoca, con evidentes efectos sociopolíticos, la idealización y la naturalización de la mujer cuidadora al resaltar el rol que ocupa la maternidad como esencia de las mujeres. Lo que se problematiza aquí es la apelación a la presencia normativa de una demanda moral dirigida a las mujeres para el cuidado, el sacrificio de sí misma y la gratuidad de sus actividades como una expresión de la feminidad.

Esa representación ideológica del sujeto madre ha incidido en la manera como el Estado delega en las mujeres el trabajo de los cuidados de forma gratuita en Latinoamérica. En Colombia, desde los años 60, un discurso desarrollista y de modernización configuró un modelo de régimen híbrido con algunas medidas redistributivas en materia 
de derechos laborales e impulso de políticas sociales en educación, vivienda, sanidad, etc., pero sin cambiar las relaciones sociales y estructuras tradicionales de dominación en amplios sectores de la economía. El Estado, al carecer de capacidad para ofrecer alternativas a los sectores más pobres, integró la creación e inversión de programas con el acompañamiento de organizaciones de mujeres que participaron políticamente alrededor de responsabilidades de género. Las mujeres fueron capacitadoras de otras mujeres y llevaron a cabo actividades de cuidado de forma solidaria de la población vulnerable - en los sectores populares, por ejemplo, las mujeres se organizaron para mejorar las viviendas y las zonas comunes de los barrios-. Esta participación "para el Estado y la sociedad significaba racionalizar un conjunto de acciones, que dispersas no podrían entregarse como complementarias a la política social; pero coordinadas sumaban activos femeninos que podían ayudar a la paz social" (Villarreal, 1994, p. 154).

Para Luna (1994), la institucionalización del maternalismo responde a los diferentes intereses que el Estado persigue; en el caso de las mujeres, es la "utilización de su capacidad como agentes sociales para el desarrollo de la comunidad y su productividad como agentes económicos domésticos" (p. 49). Esto devela una situación paradójica de inclusión y exclusión, pues, por un lado, las políticas sociales incluyen a las mujeres como objeto de la política apelando a su capacidad de agente moral para el desarrollo de actividades para el bienestar de la comunidad y en favor de la organización del Estado; pero, por otro lado, se las excluye como sujeto de poder, en el sentido en que su trabajo no significó acceso y distribución de poderes en el sistema político.

Esto se suma al carácter múltiple y contradictorio de la identidad de las mujeres trabajadoras en los cuidados por la doble presencia en el ámbito doméstico y remunerado. Arango (2011) considera que "en el trabajo de cuidado el problema de la identidad parte de la confusión entre identidad de género e identidad profesional" (p. 96), ya que este trabajo se ve atravesado por contradicciones que profundizan el trabajo precario. En ese sentido, Arango explica que el problema de la identidad se puede analizar a través de tres ejes, a saber: 1) la invisibilidad que oculta y niega todo valor social de las tareas realizadas por mujeres, representando esos deberes de madre, esposa o hija bajo la expresión del amor; 2) la servidumbre o neoservidumbre, articulada en relaciones de poder de clase, raza y etnia, que ha naturalizado la existencia de grupos sociales destinados a servir y otros, a ser servidos —así, las mujeres desplazadas 
de la ruralidad que no encuentran otra alternativa de trabajo terminan como trabajadoras domésticas, muchas veces en condiciones precarias y serviles- - 3) la profesionalización como una búsqueda de las trabajadoras de cuidados por separar "las competencias y saberes [...] de las cualidades naturales de las mujeres e identificarlos como calificaciones" (Arango, 2011, pp. 97-98).

Con esto se dilucida el significado político que implica para las mujeres el trabajo del cuidado. Estas cargas ideológicas no han impedido la inserción de las mujeres al mundo del trabajo remunerado, pero este ha estado trazado por la gratuidad, justificada por discursos de solidaridad para con la sociedad (el Estado). Tales condiciones de desigualdad refuerzan el cuidado en las mujeres en condiciones precarias.

\section{Los cuidados en el régimen de bienestar}

\section{Colombia, un régimen familista}

El trabajo de los cuidados tiene una relación con el régimen de bienestar que tengan los Estados. Los cuidados se vuelven un componente para el desarrollo social y económico y operan de manera muy diferente según el grado de bienestar del que goce cada país. Los Estados de bienestar responden a una construcción teórica sobre su capacidad mediadora en la correlación de fuerzas entre la sociedad y el mercado para asegurar la integración de clases y grupos sociales. Esto tiene como resultado la promoción de derechos sociales basados en la redistribución para reducir las desigualdades y permitir a las personas y familias mantener una buena calidad de vida independientemente de su participación en el mercado.

Lo anterior se conoce como el criterio de desmercantilización (Esping Andersen, 1993). La desmercantilización en la relación tripartita Estadomercado-familias es problematizada por el feminismo al considerar el sesgo de género que adopta dicho criterio como rasgo definitorio de los Estados de bienestar, puesto que este referente está condicionado por la experiencia vital propia de los varones y su participación en el mercado de trabajo, vinculada al estatus de trabajador y a la relación salarial, cuya representación prototípica era el varón (Campillo, 2005; Fraser, 2015; Scott, 1993).

En Latinoamérica, dicha construcción no es una realidad plena, pues se ha basado principalmente en experiencias de la Europa occidental y de países nombrados como desarrollados, mientras la región tiene unas características propias (Campillo, 2005; Mercosur, 2017). En 
Latinoamérica, la manera y el grado como se cubren y comparten las contingencias de riesgos sociales están marcados por tres características que se reflejan en los sistemas de protección social y en la relación entre Estado, mercado y familia. Existe un alto nivel de desigualdad socioeconómica, especialmente en la distribución de la riqueza, aunque con una marcada heterogeneidad entre países (Cepal, 2019), y ello conlleva la informalidad laboral, reflejada en la escasa capacidad de absorber la fuerza de trabajo y proveer remuneración de calidad, estabilidad y protección social. Por último, persiste una organización desigual de los cuidados, pues las actividades de reproducción social, proveedoras de bienestar, como el cuidado, las desempeñan principalmente las mujeres.

Desde esta perspectiva, autoras feministas latinoamericanas abordan la discusión de los regímenes de bienestar en el contexto específico de las características de la región, centrando su interés en la manera en que se moldean mutuamente las relaciones de género y los Estados a través de la familia y la división sexual del trabajo. Juliana Martínez (2008), por ejemplo, analiza los regímenes de bienestar en América Latina y las relaciones de género, destacando que la categoría de bienestar, asociada a la satisfacción de necesidades básicas requeridas para la gestión de los riesgos, no es neutral ni universal. Los riesgos varían a partir de dimensiones como la clase social y el género y son el resultado tanto de la interpretación social como del reflejo de decisiones colectivas cristalizadas en el plano de lo individual y de las políticas públicas. Así, Martínez pone en el centro del análisis las prácticas de asignación de recursos, la división sexual del trabajo y la noción de trabajo, además de la familiarización como práctica de una nueva "arquitectura del bienestar" ante las fallas o vacíos del Estado y los mercados laborales. Ella define tres tipos de regímenes en América Latina: el estatal productivista, el estatal proteccionista y el régimen familista, identificando los grados de desigualdad de género en dimensiones del mercado laboral, la política pública y la producción doméstica (Martínez, 2007; Martínez \& Voorend, 2009).

De las anteriores categorías, a Colombia se lo define en el régimen familista ${ }^{5}$. Esta denominación la marca de manera central el ámbito doméstico porque transforma a los hogares en unidades productivas que buscan compensar la falta de empleo formal y la participación estatal, se llevan a cabo prácticas

5 Este régimen lo componen dos subgrupos, el primero, con Colombia, está República Dominicana, Venezuela, Ecuador, El Salvador, Perú y Guatemala. En el otro grupo se encuentran Bolivia, Honduras, Nicaragua y Paraguay. 
productivas informales mucho más amplias y se depende en mayor medida de los ingresos, pero con más dificultades para obtenerlos. Existe, además, un patriarcado público mercantit . Es decir, hay una proporción importante de participación combinada de mujeres en el mercado laboral, tanto en sectores con altos ingresos como en los de baja remuneración. En ese sentido, "la combinación de alta participación laboral con escasa educación formal constituye una combinación fatal conducente a la subordinación pública de las mujeres" (Martínez \& Voorend, 2009, pp. 49-53), surgiendo una estructura ordenadora de las relaciones de género.

Durante los últimos treinta años, el enfoque de las políticas sociales para el acceso a programas estatales y de inversión pública en materia de cuidados ha mantenido un enfoque familista, que intensifica el trabajo de las mujeres en el interior de los hogares y en la comunidad, abaratando costos de inversión y reforzando patrones de género en el papel de cuidadoras. Por otro lado, la manera como se asigna el gasto social esencializa y despolitiza a las mujeres haciéndolas dependientes y subordinadas al Estado.

\section{Tratamiento del trabajo de los cuidados en Colombia}

La problematización sobre la importancia del trabajo reproductivo de los cuidados ha estado presente en los últimos 30 años. Durante el proceso preconstituyente, participaron grupos de mujeres dedicadas a esta labor, como el Comité de Madres Comunitarias, y se presentaron propuestas que proyectaban el trabajo doméstico como una función social de la producción y la reproducción de la fuerza de trabajo, proponiendo la garantía de seguridad social por su realización (Quintero, 2006, p. 5).

El cambio constitucional tuvo un resultado relevante en materia de derechos de las mujeres: se consagraron la igualdad de oportunidades, la abolición de toda forma de discriminación, la participación política y especial protección del Estado a mujeres cabeza de hogar. Sin embargo, si bien el derecho a la igualdad y a la no discriminación laboral fueron sancionados en la Constitución, no se materializó el trabajo de los cuidados en toda su dimensión. Esto se debe a que la Constitución de 1991 consolidó el proyecto neoliberal, pues otorgó derechos y libertades

\footnotetext{
6 Se basa en el postulado teórico de Sylvia Walby (1990) sobre variaciones de la relación patriarcal pública y privada a partir de su interactuación con diversas estructuras patriarcales. Según Walby, desde una perspectiva sistémica, un régimen de desigualdad como el género, al interactuar con ámbitos institucionales como la economía o la política, puede producir desigualdades complejas.
} 
individuales, pero, a su vez, libertades económicas amplias y promovió la privatización. En medio de esos cambios, se procuró un esquema de protección social dirigido a promover una política social viable y de bajo costo, principalmente dedicada al combate de la pobreza y una expansión del rol del mercado en la prestación de servicios sociales (Martínez, 2008; Mercosur, 2017). Esas reformas afectaron la manera como el trabajo reproductivo se articulaba con las lógicas productivas de mercado y la política social, produciendo desigualdades.

Es importante resaltar que el mercado laboral colombiano mantiene su fuerza de trabajo concentrada en sectores económicos como el de servicios, cuyo margen de productividad es menor que el de otros sectores, como el industrial. El sector de servicios tiene la mayor tasa de actividad y una de sus ramas está compuesta por los servicios comunales sociales y personales, la cual abarca actividades profesionales y técnicas en materia de cuidados, como educación, actividades en salud y el trabajo doméstico remunerado. En este aspecto la proporción de ocupación es abiertamente diferente en ambos sexos: 66,7\% en mujeres y 33,2\% en hombres (DANE, 2019c). Tal concentración de la oferta de mano de obra femenina responde a la persistencia de sesgos de género en la educación y profesionalización de disciplinas que se encuentran en el marco de los cuidados.

En cuanto al mercado de trabajo informal, existe una representación sustancial de ocupación: este trabajo concentra sectores de baja productividad y con muy poco acceso a prestaciones sociales de tipo contributivo. Por ejemplo, en el caso del trabajo doméstico remunerado, solo el $17 \%$ de las trabajadoras en hogares privados tiene acceso a la seguridad social, pues la mayoría de ellas son contratadas por días y aún reciben alimentos como parte de pago; $y$, en cuanto a los ingresos, un $62 \%$ recibe un salario mínimo o menos (UTRASD, 2020, p. 9).

De acuerdo con el panorama laboral descrito, la dimensión de la política pública en Colombia se ha caracterizado por programas estatales y de inversión con un enfoque familista, que van dirigidos a madres gestantes, subsidios a mujeres cabeza de hogar y formación de las madres comunitarias (DNP, 2019). En el caso del gasto social, por años se han atendido necesidades básicas con recursos destinados a la primera infancia y la ejecución de programas de transferencias condicionadas y apoyo económico directo, privilegiando el pago en mujeres del hogar. Esto refleja un sesgo de género, pues las transferencias condicionadas intensifican el rol de cuidadoras solamente en las mujeres, haciéndolas dependientes y subordinadas al Estado. 
Al mismo tiempo, existe una porción importante de trabajo no remunerado que atiende insuficiencias de protección social en las familias y en la comunidad, el cual se constituye en una producción doméstica gratuita dentro de las estrategias familiares en el manejo de los riesgos, bajo responsabilidad de las mujeres. Actualmente, en la distribución de los cuidados al interior de los hogares, las mujeres siguen participando e invirtiendo más horas en actividades del hogar y cuidado no remunerado ${ }^{7}$ : un $90 \%$ de las mujeres invierten hasta siete horas por día, en promedio, a diferencia de los hombres, que solo participan en un $62 \%$ e invierten apenas tres horas, según la última Encuesta Nacional de Uso del Tiempo (DANE, 2019a).

Al comparar estos cuidados con el comportamiento de la economía colombiana entre el grupo de actividades que generaron mayor valor agregado en ese año, el valor económico a precios corrientes del trabajo del cuidado no remunerado es superior (DANE, 2018). Además, el valor agregado como porcentaje del PIв de los cuidados, en contraste con actividades de cuidado remuneradas de salud, educación y servicio doméstico, es superior (DANE, 2019b). Es decir que el trabajo dentro del hogar mantiene una relación intrínseca con el sistema económico que organiza y divide sexualmente el proceso del trabajo.

Figura 1 Valor agregado y participación del pib del TDCNR

\section{Valor agregado bruto representativo de la economía (2017)}

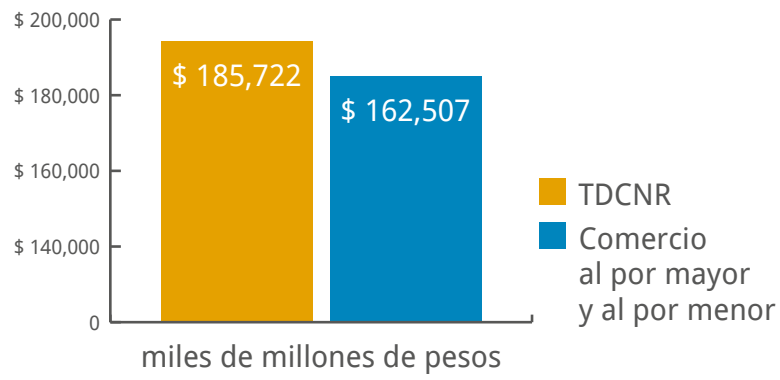

7 Dentro del Sistema de Cuentas Nacionales, que organiza y registra la información de un sector económico o social, las actividades que se atribuyen a la producción de servicios por el que no se percibe remuneración alguna no se encuentran en la frontera de la producción del sistema de cuentas nacionales. Esas actividades corresponden a trabajos de hogar y de cuidado no remunerado y trabajo voluntario. 


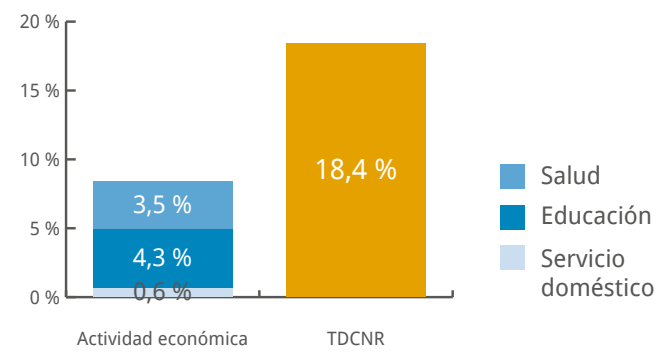

Fuente: elaboración propia, a partir de DANE (2019b).

A partir de lo anterior, se concluye que el trabajo de los cuidados no solo proporciona bienestar a los individuos, sino que también tiene una importancia en el desarrollo económico y social. El problema radica, primero, en que el Estado colombiano ha hecho responsable principalmente a las mujeres de las estrategias familiares en el manejo de los riesgos sociales, con lo cual despolitiza el conflicto social en materia de derechos como la salud, la educación y el empleo; segundo, convierte los cuidados en un problema de responsabilidad individual, y, tercero, ubica a las mujeres más pobres en un lugar de dependencia con respecto a los subsidios.

Para ilustrar esta realidad, es necesario hacer un recorrido a través de la experiencia de las madres comunitarias, trabajadoras de los cuidados, y su lucha por ser nombradas como trabajadoras y por el reconocimiento de los derechos laborales, después de desempeñar una función esencial dentro de la política pública del cuidado de la niñez colombiana.

\section{El trabajo de las madres comunitarias y las reivindicaciones judiciales}

\section{Régimen jurídico y condiciones de laborales}

Entre los años 60 y 70, se produjo una incorporación significativa de mujeres al mercado laboral colombiano en las zonas urbanas, sobre todo, en el sector terciario de los servicios domésticos, de aseo y comercio, al tiempo que aumentó el trabajo y la economía informal (Arango, 1994; Reyes \& Saavedra, 2005). Las familias de los sectores más pobres no tienen con quién dejar a sus hijos pequeños mientras trabajan, por lo que varias mujeres de los barrios se organizaron solidariamente para cuidar de niñas y niños en sus propios domicilios. Esta iniciativa consistió en la satisfacción 
de necesidades primarias de alimentación, cuidado y educación con recursos de la misma comunidad.

En 1968, se creó el Instituto Colombiano de Bienestar Familiar (ICBF), institución pública orientada a la prevención y protección integral de la primera infancia, la niñez, la adolescencia y el bienestar de las familias, en especial las más vulnerables. En 1979, el ICBF tuvo una reestructuración mediante la creación del Sistema Nacional de Bienestar Familiar, incorporando a las familias y a la comunidad como partícipes en los programas. En los años 80, coincidiendo con los primeros ajustes económicos, el programa de Gobierno modificó el modelo de atención a la infancia con la implementación de los Hogares Comunitarios de Bienestar ${ }^{8}$ (носові) para atender a la niñez entre cero y seis años de las familias más pobres del país.

Con la formalización de los diferentes hogares a través del programa носові, las mujeres cuidadoras pasaron a formar parte de la estrategia para la atención de la niñez como Madres Comunitarias. Según se describe en el Decreto 1.340 de 1995 sobre el Programa, su labor de cuidado consiste en una contribución voluntaria y su vinculación no tiene carácter laboral alguno:

La vinculación de las madres comunitarias, así como la de las demás personas y organismos de la comunidad, que participen en el programa de "Hogares de Bienestar", mediante su trabajo solidario, constituye contribución voluntaria, por cuanto la obligación de asistir y proteger a los niños, corresponde a los miembros de la sociedad y la familia; por consiguiente, dicha vinculación no implica relación laboral con las asociaciones u organizaciones comunitarias administradoras del mismo, ni con las entidades públicas que en él participen (énfasis añadido).

Además, se establece un perfil para ser madre comunitaria, a partir del cual se demandan una serie de cualidades y condiciones, como poseer vivienda adecuada, aceptar la vinculación como solidaria, tener un mínimo de cuatro años de educación básica, y un reconocido comportamiento social y moral (ICBF, 1996, art. 5).

8 La Ley 89 de 1988 describe los носов с соmo espacios para atender las necesidades básicas de nutrición, salud, protección y desarrollo individual y social de los niños de los estratos sociales pobres del país, con la participación de las familias y los vecinos y utilizando un gran porcentaje de los recursos locales. 
Figura 2. Operación, características y financiación del programa HосовI

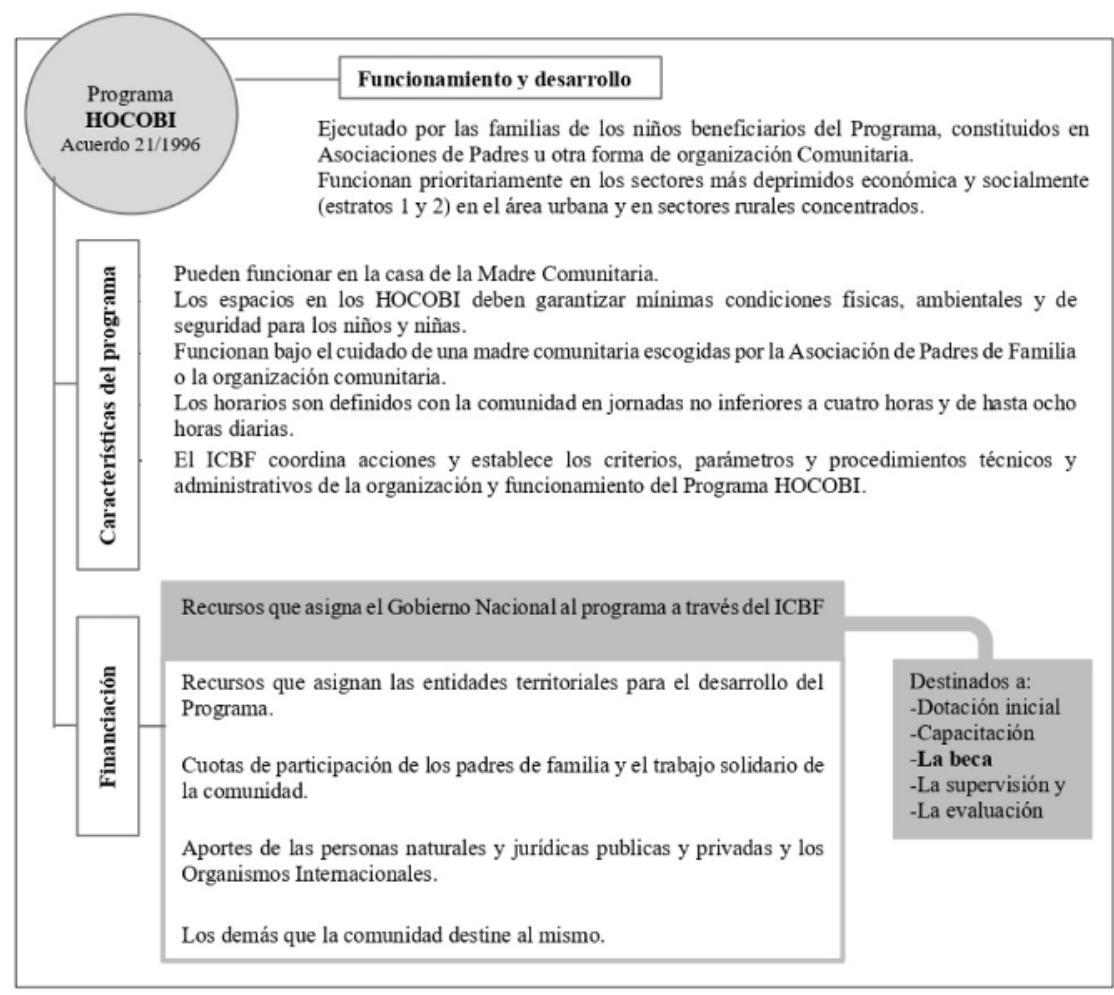

Fuente: elaboración propia, a partir de ICBF (1996).

Bajo la figura de la contribución solidaria, las madres comunitarias no recibían remuneración en términos laborales; sin embargo, se les destinaba una bonificación llamada beca, que consistía en la "reposición de dotación, aseo y combustible, raciones, material didáctico [...] y apoyo para servicios públicos" (ICBF, 1996, art. 4). Hasta el 2012, la bonificación o beca que recibieron las madres comunitarias para atender hasta 14 niños fue del $70 \%$ del salario mínimo legal vigente. En cuanto a la protección social en salud, su vinculación y cotización la asumen las madres comunitaria con el aporte del $4 \%$ de su bonificación y la cuota mensual de participación de los padres, mientras el Estado se compromete a pagar el valor restante $(8.5 \%)$ a través de un régimen especial (Congreso de la República de Colombia, Ley 509, 1999). En materia pensional, el marco legal ${ }^{9}$ establece $^{-}$ que las madres comunitarias hacen sus aportes en un $20 \%$ al Sistema 
General de Pensiones y el Estado "subsidia" el $80 \%$ a través del Fondo de Solidaridad Pensional ${ }^{10}$ (FSP), cumpliendo los requisitos de cualquier trabajador en materia de edad (57 años) y acumulación de semanas cotizadas (mínimo de 1.300) para acceder a la pensión. En realidad, muchas mujeres no alcanzan a cumplir el último requisito y se las prioriza ${ }^{11}$ legalmente con el acceso al subsidio de Subcuenta de Subsistencia.

En el 2009, se crearon los Beneficios Económicos Periódicos (BEPs) como parte de los Servicios Sociales Complementarios para la protección a la población de la tercera edad de más escasos recursos con el fin de garantizar un ingreso periódico hasta el fallecimiento, sin constituirse en una pensión. En el 2015, se les permitió a las madres comunitaria vincularse al servicio complementario de BEPS y trasladar el porcentaje del subsidio, cuando no hayan reunido los requisitos para acceder a la pensión o se encuentren lejos de alcanzarlos ${ }^{12}$ (Presidencia de la República de Colombia, Decreto 1.833, 2016, art. 4).

Dadas las condiciones de trabajo que las rigen y por hacer parte de los sectores en condiciones socioeconómicas precarias, las madres comunitarias acceden de forma prioritaria a beneficios especiales como, por ejemplo, los subsidios familiares de vivienda de interés social urbano de las cajas de compensación familiar ${ }^{13}$. En Bogotá, el costo de los servicios públicos domiciliarios en los носов es asimilados a estrato socioeconómico 1, garantizando el cubrimiento de necesidades básicas y subsidiado por los estratos más altos.

Desde esta perspectiva, el régimen jurídico del trabajo de las madres comunitarias obedece, en realidad, a una relación de subordinación, dependencia y explotación laboral en la provisión de trabajo barato y precario. La negación de un salario mínimo y de subsidios de seguridad social y pensión, así como la de beneficios especiales, neutraliza el potencial emancipador de este trabajo y traslada la mirada a un enfoque

10 El fsp es una cuenta especial de la Nación, adscrita al Ministerio del Trabajo. Según lo establecido en el artículo 13, literal i de la Ley 100 de 1993 y el artículo 2.2.14.1.1 del Decreto 1.833 del 2016, está destinado a ampliar la cobertura mediante el subsidio a los grupos de población que, por sus características y condiciones socioeconómicas, no tienen acceso a los sistemas de seguridad social y carecen de capacidad para efectuar la totalidad del aporte. (Ministerio del Trabajo de Colombia, s. f.).

11 Señalado en el artículo 164 de la Ley 1.450 del 2011 (Plan Nacional de Desarrollo, 2010-2014).

12 El Decreto 387 del 2018 reglamentó dentro de los grupos poblacionales destinatarios del beneficio a las madres comunitarias.

13 la Ley 1.537 del 2012 prioriza los subsidios de vivienda para los hogares cuyo miembro principal sea una madre comunitaria vinculada a los programas de hogares comunitarios. 
asistencialista de medidas paliativas, en vez de establecer garantías laborales, con lo cual se reproducen desigualdades de género y de clase.

\section{LA PUGNA POR NOMBRAR LOS CUIDADOS COMO TRABAJO}

La nueva Constitución consagró disposiciones importantes en materia de género y ha sido una herramienta de lucha para la materialización de derechos por la vía jurisprudencial, concentrada "en los jueces de la Corte Constitucional como interlocutor[es] legítimo[s] de las denuncias de inequidad y discriminación” (Buchely, 2014, p. 92), por lo que, en concreto, es un canal reivindicativo para obtener victorias jurídicas y, al tiempo, abrir escenarios para el desarrollo de una agenda de género inimaginable sin el cambio constitucional.

Sin embargo, los reclamos de las madres comunitarias se han encontrado con una doctrina constitucional que excusa el examen del problema sobre la realidad del trabajo de los cuidados y sigue interpretando una distinción entre los ámbitos público y privado, motivando diferencias legales que discriminan a las mujeres. Desde 1992, las mujeres trabajadoras en el programa носов han problematizado su relación con el Estado poniendo de presente la realidad sobre la naturaleza de su vinculación, y solo hasta el 2012 los pronunciamientos abordaron el valor del trabajo reproductivo de las madres comunitarias al servicio de la política pública de infancia.

A través de una acción de tutela, la Corte Constitucional reconoce, bajo un régimen especial, que las madres comunitarias son trabajadoras y que, por tanto, negar la retribución del salario mínimo constituye una discriminación. Por ello, ordenó al ICBF adoptar de medidas para asegurar que las madres comunitarias de tiempo completo devenguen "al menos" un salario mínimo completo (Corte Constitucional de Colombia, Sala Octava de Revisión, Sentencia T-628, 2012, numeral 63 a 65). No obstante, la Corte continuó sosteniendo un discurso ambiguo que no resolvía la relación de trabajo de las Madres Comunitarias con el Estado. Nombró su régimen jurídico como de carácter especial por tener características propias de trabajo subordinado (la limitación de jornada máxima laboral) y específicas del trabajo independiente, con lo cual lo tipificó como un "intermedio entre trabajo subordinado e independiente" (Corte Constitucional de Colombia, Sala Octava de Revisión, Sentencia T-628, 
2012, numeral 63). Esta respuesta ilustra la dificultad de ubicar el trabajo de cuidados como trabajo dentro del sector público.

Por orden del fallo del 2012, el Gobierno, a través de la Ley de Reforma Tributaria (Congreso de la República de Colombia, Ley 1.607, 2012), se impuso la beca de las Madres Comunitarias en un salario mínimo, de manera progresiva entre el 2013 y el 2014, hasta conseguir formalizarlas laboralmente. El artículo 36 estipuló que se diseñarían y adoptarían las modalidades de vinculación contractual sin que esto implicara otorgarles la calidad de funcionarias públicas. Sin embargo, en esos años muchas trabajadoras aún no eran vinculadas laboralmente, por lo que el sindicato sinTRACIHOBI presentó un pliego de peticiones al Gobierno, reclamando la contratación laboral a término indefinido no tercerizado y la pensión digna a las madres comunitarias mayores y enfermas que no podían seguir laborando. En respuesta, se reglamentó, a partir del artículo 36 de la Ley de Reforma Tributaria, el Decreto 289 del 2014, que estableció como modalidad de contratación de las madres comunitarias el contrato de trabajo suscrito con las entidades administradoras del programa носові, señalándolas como únicas empleadoras y obligadas laborales, mientras que el ICBF se encargaría de la inspección, vigilancia y supervisión. Este Decreto enfatiza que las madres comunitarias no tendrán la calidad de servidoras públicas y el ICBF no tendrá responsabilidad solidaria patronal alguna.

Este último marco legal cerraba la discusión sobre la forma de vinculación contractual de las madres comunitarias como trabajadoras del cuidado de la niñez dentro de un programa del Estado. Pero, aunque formalmente parecía resuelto el problema sobre la existencia de una relación laboral, en la práctica, la regulación social del trabajo de las madres comunitarias no daba respuesta plena a las labores de cuidado ejercidas. El reconocimiento de su labor como "trabajo" no amparó los derechos de su trabajo para el Estado por más de 30 años.

En 2017, un nuevo fallo de la Sala Octava de Revisión de la Corte Constitucional, por cuenta de una demanda de 106 madres comunitarias, cambiaba la interpretación socio-jurídica sobre la labor de cuidados de las cuidadoras y establecía una nueva doctrina constitucional al considerar la existencia de una relación laboral con el ICBF (Corte Constitucional de Colombia, Sala Octava de Revisión, Sentencia T-480, 2016, numeral 168).

La Sala Octava consideró que se configuró durante décadas una defraudación de derechos laborales contra las madres comunitarias al ser el ICBF el ejecutor de diversas estrategias jurídicas y actos discriminatorios 
que ocultaban un contrato real, produciendo "múltiples actos y manifestaciones que contenían o se ajustaron a una ideología diferenciada en razón de género" (Corte Constitucional de Colombia, Sala Octava de Revisión, Sentencia T-480, 2016, numeral 168.4). Esta situación recrudeció la discriminación al dirigirse contra un sector deprimido socialmente, sin garantías derivadas del derecho al trabajo, en situación económica precaria y, en su mayoría, constituido por mujeres de la tercera edad o en malas condiciones de salud. Ante esto, se declaró la existencia de un contrato real y se obligó a reconocer y pagar salarios, prestaciones sociales y aportes pensionales.

Esta decisión abría el camino para que miles de madres comunitarias reclamaran el mismo derecho, y, con ello, el ICBF adeudaría salarios desde 1988, lo que superaba hasta tres veces su presupuesto - para el 2017, era de seis billones de pesos- La entidad estatal no dudó en solicitar a la Sala Plena la nulidad del fallo, alegando el desconocimiento de la línea jurisprudencial precedente, que reiteraba la no vinculación laboral de las MADREs COMUnitarias hasta antes del Decreto $289 \mathrm{del}$ 2014. Efectivamente, mediante un Auto, la Sala Plena estudió la decisión de la Sala Octava por presunta transgresión al debido proceso y decidió anular el fallo y admitir que sí existía providencia anterior vinculante al caso, que no podía ser desconocida dentro de la decisión. Por tanto, revocó la sentencia y solo reconoció el derecho a la seguridad social por "condiciones de vulnerabilidad", obligando a la transferencia del subsidio pensional en un $100 \%$ del total de las cotizaciones faltantes para las 106 madres comunitarias solicitantes (Corte Constitucional de Colombia, Sala Plena, Auto-186, 2017).

Esta decisión implicó un retroceso y reforzó el carácter asistencialista con que se asume el problema laboral de las cuidadoras. El reconocimiento se limitó a la política social por las condiciones de vulnerabilidad, bajo prerrogativas de ser "titulares de prestaciones asistenciales" en seguridad social (Corte Constitucional de Colombia, Sala Plena, Auto186, 2017, numeral 13.2). El Auto orientó la discusión a una consideración meramente fiscal ante la imposibilidad del ICBF de asumir los costos económicos y su posible quiebra, pero soslayó el problema en términos de la supremacía de los derechos constitucionales de las madres comunitarias.

Luego, se produjo una segunda nulidad, que dejó sin efecto el amparo en materia de seguridad social que se había otorgado, por la 
vulneración al debido proceso en la indebida conformación del contradictorio ${ }^{14}$, todo porque no se vinculó a dos entidades del Estado" ${ }^{15}$, ya que lo "ordenado al Fondo de Solidaridad Pensional en el Auto 186 de 2017 [desbordaba] el deber legal que le [fue] impuesto" (Corte Constitucional de Colombia, Sala Plena, Auto-217, 2018), lo cual frenó el reconocimiento al subsidio pensional del $100 \%$ a las 106 accionantes. Las nulidades sobrevenidas por el mayor órgano judicial suponen la pérdida del reconocimiento de las debidas garantías salariales y pensionales para las Madres Comunitarias.

En un nuevo precedente de unificación (Corte Constitucional de Colombia, Sala Plena, Sentencia SU-079, 2018), en reemplazo de la sentencia T-480 de 2016, la Corte hizo un recuento jurisprudencial de los últimos años, dejando constancia de que las actividades ejercidas por las madres comunitarias no supusieron relación laboral alguna y solo a partir del 2014, con la expedición del Decreto 289, fueron vinculadas laboralmente bajo contrato de trabajo con las entidades administradoras del programa, señalando que

Si bien [...] la mayoría de las accionantes efectivamente se desempeñaron de forma permanente o periódica como madres comunitarias [...] lo cierto es que el ICBF no está llamado a responder por los derechos fundamentales por ellas invocados, pues ha sido la ley y el reglamento, quienes han establecido las características del régimen jurídico de los hogares comunitarios y sustitutos de bienestar (SU-079, 2018, numeral 35, énfasis añadido).

Tampoco fue admitida la solicitud de pago de los aportes por parte de ICBF en nombre del deber correlativo previsto en la norma, que implica el cumplimiento de requisitos de ley para acceder al programa de Subsidio al Aporte a Pensión. La Corte revocó las decisiones proferidas a este respecto por Tribunales en el año inmediatamente anterior, concluyendo que las entidades no tenían la obligación de reconocer acreencias laborales ni pago de aportes a pensión.

14 Es causal de nulidad el que la decisión se produzca sin la debida notificación a todas las partes, lesionando garantías procesales, en especial el derecho a la contradicción.

15 Al Ministerio del Trabajo, entidad a la cual se encuentra inscrito el Fondo de Solidaridad Pensional y al Consorcio Colombia Mayor, por ser la entidad que administra los recursos públicos que se encuentran en el Fondo de Solidaridad Pensional. 
Figura 3. Desarrollo de los principales antecedentes jurisprudenciales vinculantes de la Corte Constitucional colombiana

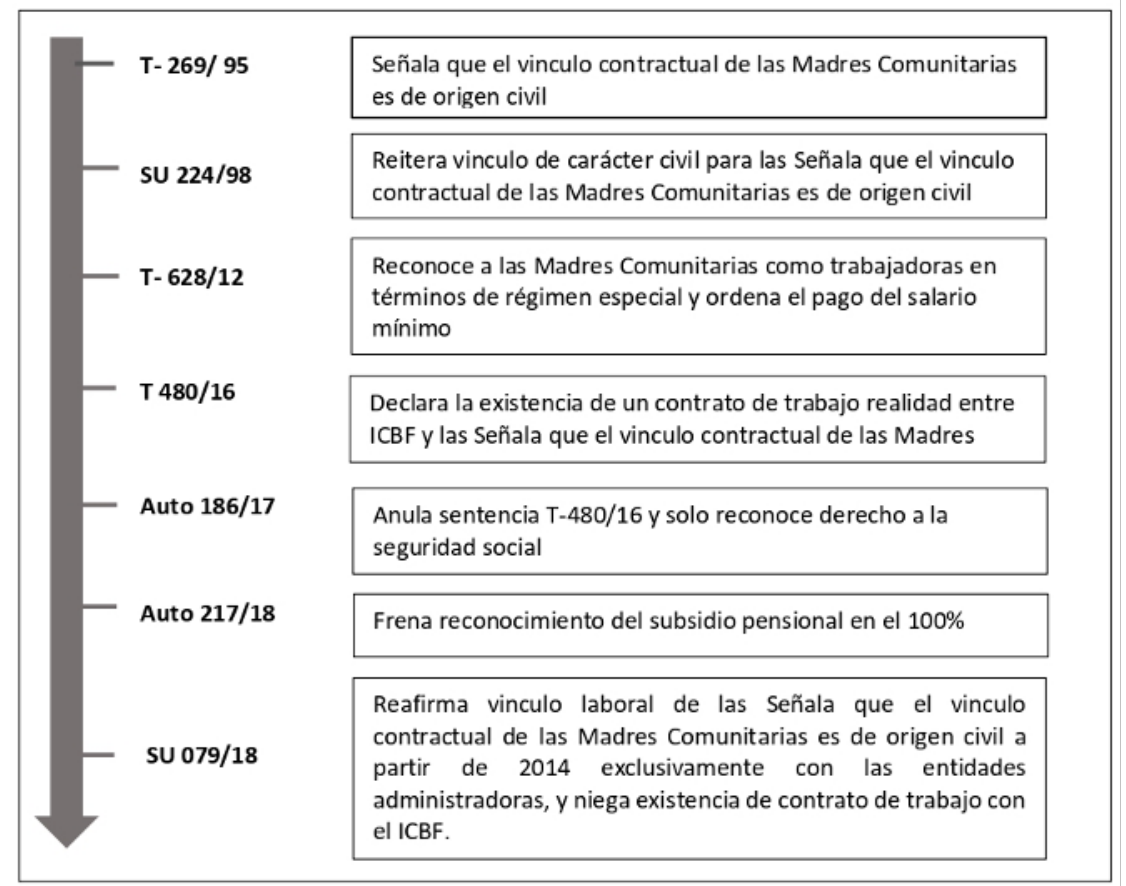

Fuente: elaboración propia, a partir de información tomada de la relatoría la Corte Constitucional de Colombia.

El problema del reconocimiento de la relación laboral y las garantías de las madres comunitarias ha sido objeto, desde hace varios años, por parte del Congreso de la República. Sin embargo, se han presentado diez iniciativas sobre el tema, de las cuales ocho han sido archivadas o retiradas por su autor, y actualmente se encuentra un proyecto de ley vigente.

El nuevo proyecto de Ley 127 del 2015 tiene por objeto fijar las reglas de la relación laboral de las madres comunitarias y establecer de manera clara sus derechos laborales. Inicialmente, proponía la vinculación contractual en forma directa con el ICBF o mediante la contratación de organizaciones, así como la garantía contractual con vocación de estabilidad laboral, el contrato a término indefinido y la existencia de un subsidio permanente a la vejez. Pero, tras los debates en ambas cámaras del Congreso de la República, en diciembre del 2017, el Gobierno formuló objeciones al proyecto por razones de inconstitucionalidad, basado en la 
competencia gubernamental exclusiva y la conveniencia en materia de sostenibilidad fiscal.

La Corte Constitucional (Sala Plena, Auto de insistencia probatoria OG-158, 2019) admitió y consideró fundadas las objeciones y devolvió el proyecto de Ley al Congreso para que se subsanara el vicio de forma y tramitara nuevamente la iniciativa. Después de las correcciones, el proyecto precisó que el vínculo laboral contractual solamente fuera con las organizaciones del programa y su remuneración, no inferior al salario mínimo, con derecho a un subsidio permanente de vejez equivalente al $95 \%$ del salario mínimo, si ha laborado durante 20 años o más, o uno proporcional al tiempo laborado, en el caso de haberlo hecho menos de 20 años y más de 10. Actualmente, se encuentra vigente y pendiente para revisión constitucional y sanción de la rama ejecutiva.

Esta iniciativa es la que más lejos ha llegado, en comparación con las otras nueve que se han presentado desde el 2001. Intenta amparar, en favor de las madres comunitarias, el trabajo de los cuidados bajo una regulación y garantizar una protección para la vejez de las mujeres que por largo tiempo han trabajado en los cuidados. Pero deja en evidencia las dificultades que ha tenido la institucionalidad en el replanteamiento de las categorías jurídicas para legislar en materia de derechos de las mujeres.

\section{CONCLUSIONES}

El género es un elemento constitutivo de las relaciones sociales, en el cual se articulan ejes de subordinación, como la clase y la raza; en últimas, es un principio ordenador de la estructura económica de la sociedad capitalista. Por tanto, la categoría de trabajo, que organiza, clasifica y diferencia las esferas productiva e improductiva, es una muestra de que no es una categoría ni objetiva ni neutral respecto al género.

La disputa por nombrar los cuidados como trabajo cuestiona los conceptos hegemónicos de lo que es productivo y visibiliza los trabajos para la sostenibilidad de la vida, que son los que justamente posibilitan la producción económica. La participación de las mujeres en el mercado laboral, el desarrollo de trabajos de cuidados remunerados y la presencia de nuevos actores sociales impulsan la necesidad de reconceptualizar la categoría trabajo. Pensar el trabajo de las madres comunitarias revela el carácter múltiple y contradictorio que tiene el trabajo de los cuidados en Colombia por el hecho de desarrollarse en el ámbito doméstico 
y exigir una remuneración. Por ello, la orientación maternalista y el tratamiento del trabajo como un vínculo de solidaridad ha dificultado el reconocimiento de garantías laborales.

A pesar del esfuerzo por problematizar el trabajo de los cuidados desde una perspectiva de género, las decisiones de la Corte Constitucional siguen manifestando una posición tradicional entre el ámbito público y el privado frente a la supremacía de los derechos constitucionales para la tutela de los derechos laborales y la protección social de las madres comunitarias, por lo que prioriza una política pública de cuidados a la primera infancia sobre el trabajo de las cuidadoras. Con esto se refuerzan los mandatos socioculturales que esencializan a las mujeres en relación con la labor de cuidar, agudizando la explotación y discriminación laboral.

La desvalorización del trabajo de los cuidados de las madres comunitarias también radica en la persistencia del Estado por hacerlas dependientes y subordinadas. Se instrumentaliza su trabajo con la incorporación "prioritaria” de subvenciones y beneficios de políticas sociales bajo un "régimen especial", pero estos ocultan el amparo de derechos laborales, como la remuneración y las prestaciones sociales, con lo cual se profundiza la desigualdad y la precariedad.

No obstante, la Constitución de 1991 abrió escenarios para una agenda de género con respecto a los cuidados. La reivindicación de los derechos de las mujeres por la vía de la jurisprudencia ha replanteado el desarrollo de la doctrina constitucional tradicional al convertir en agenda pública el trabajo de los cuidados. La lucha de las Madres Comunitarias por nombrar el trabajo de los cuidados al servicio del Estado y demandar el reconocimiento de sus derechos laborales supone una forma en que las mujeres inciden para que el discurso constitucional aborde asuntos de género y se traduzca en un discurso y unas prácticas de derechos.

\section{REFERENCIAS}

Arango, L. G. (1994). Industria textil y saberes femeninos. Historia Crítica, (9), 4449. https://doi.org/10.7440/histcrit9.1994.06

Arango, L. G. (2011). El trabajo de cuidado: ¿Servidumbre, profesión o ingeniería emocional? En L. G. Arango, \& M. Pascale (comps.), El trabajo y la ética del cuidado (pp. 91-107). La Carreta Social.

Arendt, H. (1993). La condición humana. Paidós.

Arruza, C. (2014, 2 de septiembre). Remarks on Gender. Viewpoint Magazine. https://www.viewpointmag.com/2014/09/02/remarks-on-gender/ 
Artiaga Leiras, A. (2015). Producción politica de los cuidados y de la dependencia: Políticas públicas y experiencias de organización de los cuidados [tesis doctoral, Universidad Complutense de Madrid]. Repositorio institucional. https:// eprints.ucm.es/id/eprint/32816/

Buchely, L. F. (2014). Género y constitucionalismo. Una mirada feminista al derecho constitucional colombiano. Ciencia Politica, 9(18), 83-107. https:// revistas.unal.edu.co/index.php/cienciapol/article/view/52309

Burgos, G., Carvajal, J., Estrada, J., Flórez, M., León, H., \& Moreno, S. (2009). El BID en América Latina: 50 años reproduciendo la desigualdad. Instituto Latinoamericano de Servicios Legales Alternativos.

Campillo, A. (2003). ¿Quién gobierna mi vida? El pensamiento de Michel Foucault. En E. M. Albert, \& J. Sáez (coords.), Pensadores de ayer para problemas de hoy: Teóricos de las Ciencias Sociales (pp. 127-146). Editorial UIC.

Campillo, I. (2005, 21 de septiembre). La ciudadanía social. Perspectivas y propuestas feministas [conferencia]. VII Congreso de la Asociación Española de Ciencia Política y de la Administración, Madrid, España. http://pmayobre.webs. uvigo.es/pop/archi/profesorado/monica_lopez/campillo.pdf

Comisión Económica para América Latina y el Caribe (Cepal) (2019). Panorama social de América Latina, 2018. Naciones Unidas.

Congreso de la República de Colombia (1988, 29 de diciembre). Ley 89 de 1988, por la cual se asignan recursos al Instituto Colombiano de Bienestar Familiar y se dictan otras disposiciones. Diario Oficial n. ${ }^{\circ}$ 38.635. https://www.icbf.gov.co/sites/ default/files/ley_89_de_1988.pdf

Congreso de la República de Colombia (1999, 30 de julio). Ley 509 de 1999, por la se disponen unos beneficios en favor de las Madres Comunitarias en materia de Seguridad Social y se otorga un Subsidio Pensional. Diario Oficial n. ${ }^{\circ} 43.653$.

Congreso de la República de Colombia. (2011, 16 de junio). Ley 1.450 del 2011, por la cual se expide el Plan Nacional de Desarrollo. Diario oficial n. ${ }^{\circ} 48.102$.

Congreso de la República de Colombia (2012, 20 de junio). Ley 1.537 del 2012, por la cual se dictan normas tendientes a facilitar y promover el desarrollo urbano y el acceso a la vivienda y se dictan otras disposiciones. Diario Oficial n. ${ }^{\circ} 48.467$.

Congreso de la República de Colombia (2012, 26 de diciembre). Ley 1.607 del 2012, por la cual se expiden normas en materia tributaria y se dictan otras disposiciones. Diario Oficial n. ${ }^{\circ} 48.655$.

Corte Constitucional de Colombia, Sala Octava de Revisión. (2012, 10 de agosto). Sentencia T-628/12 [MP Humberto Sierra Porto].

Corte Constitucional de Colombia, Sala Octava de Revisión. (2016, 1 de septiembre). Sentencia T-480/16 [mp Alberto Rojas Ríos].

Corte Constitucional de Colombia, Sala Plena. (2017, 17 de abril). Auto-186/17 [Ms Alberto Rojas Ríos].

Corte Constitucional de Colombia, Sala Plena (2018, 11 de abril). Auto-217/18 [ms Alberto Rojas Ríos].

Corte Constitucional de Colombia, Sala Plena (2018, 9 de agosto). Sentencia SU079/18 [MP José Fernando Reyes Cuartas]. 
Corte Constitucional de Colombia, Sala Plena (2019, 17 de octubre). Auto de insistencia probatoria OG-158/19 [Ms Alejandro Linares Cantillo].

Departamento Administrativo Nacional de Estadística (DANE) (2018, 30 de agosto). Cuenta Satélite de Economía del Cuidado CSEC 2017. https://www.dane.gov. co/files/investigaciones/boletines/cuentas/ec/Bol_CS_Econo_cuidado_ TDCNR_2017.pdf

Departamento Administrativo Nacional de Estadística (DANE) (2019a, 12 de mayo). Encuesta Nacional de Uso del Tiempo ENUT. https://www.dane.gov.co/index.php/ estadisticas-por-tema-2/pobreza-y-condiciones-de-vida/encuesta-nacionaldel-uso-del-tiempo-enut

Departamento Administrativo Nacional de Estadística (dane) (2019b, 29 de agosto). Cuenta Satélite de Economía del Cuidado (CSEC) 2017. https://www.dane. gov.co/files/investigaciones/boletines/cuentas/ec/Bol_CS_Econo_cuidado_ CPEC_2017_prov.pdf

Departamento Administrativo Nacional de Estadística (DANE) (2019c, 10 de mayo). Mercado laboral-empleo y seguridad social. http://www.dane.gov.co/index.php/ estadisticas-por-tema/mercado-laboral/empleo-informal-y-seguridad-social

Departamento Nacional de Planeación (DNP) (2019d, 25 de mayo). Bases del Plan nacional de Desarrollo 2018-2022. Pacto por Colombia Pacto por la equidad. https://www.dnp.gov.co/Plan-Nacional-de-Desarrollo/Paginas/Bases-delPlan-Nacional-de-Desarrollo-2018-2022.aspx

Esping Andersen, G. (1993). Los tres mundos del Estado del bienestar. Edicions Alfons el Magnànim.

Federici, S. (2015). Calibán y la bruja. Mujeres, cuerpo y acumulación originaria. Tinta y Limón.

Foucault, M. (2002). Vigilar y castigar: nacimiento de la prisión. Siglo XXI.

Fraisse, G. (2003). Los dos gobiernos: La familia y la ciudad. Cátedra.

Fraser, N. (2006). La justicia social en la era de la política de identidad: Redistribución, reconocimiento y participación. En N. Fraser \& A. Honneth, ¿Redistribución o reconocimiento? (pp. 19-51). Morata.

Fraser, N. (2014). Tras la morada oculta de Marx. New Left Revew, (86), 57-76.

Fraser, N. (2015). Las contradicciones del capital y los cuidados. New Left Review, (100), 111-131.

Fuente, M. de la (2015). Ideas de poder en la teoría feminista. Revista Española de la Ciencia Política, (39), 173-193.

Instituto Colombiano de Bienestar Familiar (ICBF) (1996, 23 de abril). Acuerdo 21 de 1996, por el cual se dictan lineamientos y procedimientos técnicos y administrativos para la organización y funcionamiento del Programa Hogares Comunitarios de Bienestar. https://www.icbf.gov.co/cargues/avance/docs/ acuerdo_icbf_0021_1996.htm

Luna, G. (1994). Historia, género y política. En G. Luna, \& N. Villarreal, Historia, Género y Política Movimiento de mujeres y participación política en Colombia 1930-1991 (pp. 19-58). Edición Seminario Interdisciplinar Mujeres y Sociedad. 
Martín Palomo, M. T. (2011). "Domesticar" el trabajo: una reflexión a partir de los cuidados. En L. G. Arango, \& M. Pascale (coords.), El trabajo y la ética del cuidado (pp. 67-85). La Carreta Social.

Martínez, J. (2007). Regímenes de bienestar en América Latina. Fundación Carolina -CeALCI.

Martínez, J. (2008). Domesticar la incertidumbre en América Latina: mercado laboral, política social y familias. Editorial Universidad de Costa Rica.

Martínez, J., \& Voorend, K. (2009). Sistemas de patriarcado y regímenes de bienestar en América Latina ¿̇Una cosa lleva a la otra? Fundación Carolina - CeALCI.

Méda, D. (2007). ¿Qué sabemos sobre el trabajo? Revista de Trabajo, 3(4), 17-32.

Mercado Común del Sur (Mercosur) (2017). Bienestar y protección social en América Latina. Instituto Social del Mercosur.

Ministerio del Trabajo de Colombia (s.f.). Fondo de Solidadridad Pensional. Fondo de Solidaridad Pensional. https://www.fondodesolidaridadpensional.gov.co/ fondo-de-solidaridad/que-es-el-fondo-de-solidaridad-pensional.html

Organización Internacional del Trabajo (оIт). (2018). El trabajo de cuidados y los trabajadores del cuidado: Para un futuro con trabajo decente. ort. https://www. ilo.org/wcmsp5/groups/public/---dgreports/---dcomm/---publ/documents/ publication/wcms_633168.pdf

Paredes, J. (2008). Hilando fino desde el feminismo comunitario. Mujeres del mundo "Babel". http://mujeresdelmundobabel.org/files/2013/11/Julieta-ParedesHilando-Fino-desde-el-Fem-Comunitario.pdf

Pérez Orozco, A. (2017). Subversión feminista de la economía: Aportes para un debate sobre el conflicto capital-vida. Traficantes de Sueños.

Presidencia de la República de Colombia (1995, 10 de agosto). Decreto 1.340 de 1995, por el cual se dictan disposiciones sobre el desarrollo del Programa Hogares Comunitarios de Bienestar. Diario Oficial 41.960.

Presidencia de la República de Colombia (2016, 10 de noviembre). Decreto 1.833 de 2016, por el cual se compilan las normas del Sistema General de Pensiones. Diario Oficial 50.053.

Quintero, B. (2006). Las mujeres colombianas y la Asamblea Nacional Constituyente de 1991: participación e Impactos. En Reformas Constitucionales y Equidad de Género: Informe final del Seminario Internacional (pp. 269-285). Cepal.

Reyes, A. C., \& Saavedra, M. C. (2005). Mujeres y trabajo en Antioquia durante el siglo $x x$. Ediciones Escuela Nacional Sindical.

Scott, J. (1993). La mujer trabajadora en el siglo xıx. En G. Duby, \& M. Perrot, Historia de las mujeres. El siglo XIX (vol. 4, pp. 425-461). Taurus.

Serrano, A., \& Prieto, C. (2013). Los cuidados entre el trabajo y la vida. Cuaderno de Relaciones Laborales, 31(1), 11-16.

Villarreal, N. (1994). Flujos y reflujos en los movimientos de mujeres, 1958-1975.

En L. Luna, \& N. Villarreal, Historia, Género y Política Movimiento de mujeres y participación política en Colombia 1930-1991 (pp. 147-170). Edición Seminario Interdisciplinar Mujeres y Sociedad. 
Unión de Trabajadoras Afrocolombianas del Servicio Doméstico (UTRASD) (2020).

Hacer visible lo invisible del trabajo remunerado del hogar. Medellín.

Walby, S. (1990). Theorizing Patriarchy. Basil Blackwell.

Weber, M. (1969). Economía y sociedad. Esbozo de sociología comprensiva. FCE. 